\title{
Síndrome de Lemmel como etiología de pancreatitis aguda
}

\author{
Lemmel's syndrome as etiology of acute pancreatitis \\ Luis Rubén Sosa Flores, ${ }^{\star}$ Pastor Israel Díaz Hernández, ${ }^{\star}$ María Andrea Peñaloza Posada ${ }^{\ddagger}$ \\ * Departamento de Cirugía General. \\ ‡ Departamento de Gastroenterología y Endoscopía Gastrointestinal. \\ Hospital Regional de Alta Especialidad Ixtapaluca. Estado de México, México.
}

\section{RESUMEN}

El síndrome de Lemmel se define como la presencia de ictericia con patrón obstructivo en pacientes con divertículos duodenales, en ausencia de coledocolitiasis o neoplasias. Se pueden observar hasta en $22 \%$ de la población, lo cual aumenta con la edad. La importancia radica en que la mayoría de los divertículos duodenales se encuentran en la segunda porción del duodeno, adyacentes al ámpula de Vater 0 a una distancia de 2-3 cm, por lo que no sólo causan compresión mecánica del conducto biliar, sino que también inducen la disfunción del esfínter de Oddi. Presentamos el caso de un hombre de 51 años de edad, con dolor y cuadro clínico de pancreatitis aguda; se descartaron las causas más frecuentes de pancreatitis aguda y se realizó colangiopancreatografía retrógrada endoscópica, en la cual se evidenció el divertículo duodenal.

Palabras clave: Síndrome de Lemmel, divertículo duodenal, pancreatitis.

\section{ABSTRACT}

Lemmel's syndrome is defined as the presence of jaundice with an obstructive pattern in patients with duodenal diverticula, in the absence of choledocholithiasis or neoplasms. They can be observed in up to $22 \%$ of the population, which increases with age. The importance lies in the fact that most duodenal diverticula are found in the second portion of the duodenum, adjacent to the ampulla of Vater or at a distance of 2-3 $\mathrm{cm}$, so they not only cause mechanical compression of the bile duct, but also induce sphincter of Oddi dysfunction. We present the case of a 51-year-old male patient, with pain in the clinical picture of acute pancreatitis, where the most frequent cases of acute pancreatitis were ruled out, undergoing endoscopic retrograde cholangiopancreatography, where the duodenal diverticulum was evidenced.

Keywords: Lemmel syndrome, duodenal diverticulum, pancreatitis.

\section{INTRODUCCIÓN}

El síndrome de Lemmel se define como la presencia de ictericia con patrón obstructivo en pacientes con divertículos duodenales, en ausencia de coledocolitiasis o neoplasias. ${ }^{1-3}$

Existen pocos casos reportados en la literatura y el primero fue publicado por Lemmel en $1934 .^{2}$

Sólo $5 \%$ de los divertículos duodenales presentan síntomas; ${ }^{4,5}$ sin embargo, se pueden observar hasta en
$22 \%$ de la población, lo cual aumenta con la edad hasta $27 \% .^{2,4}$ La importancia radica en que la mayoría de los divertículos duodenales se encuentran en la segunda porción del duodeno, adyacentes al ámpula de Vater o a una distancia de $2-3 \mathrm{~cm}, 1,4,5$ por lo que no sólo causan compresión mecánica del conducto biliar, sino que también inducen la disfunción del esfínter de Oddi con estasis de bilis secundaria y reflujo duodenal hacia el colédoco como fisiopatología de colangitis.

\section{Recibido: 18/01/2021. Aceptado: 02/02/2021 \\ Correspondencia: Luis Rubén Sosa Flores \\ E-mail: rubensf2693@gmail.com}

Citar como: Sosa FLR, Díaz HPI, Peñaloza PMA. Síndrome de Lemmel como etiología de pancreatitis aguda. Rev Mex Cir Endoscop. 2020; 21 (3): 163-165. https://dx.doi.org/10.35366/99842 


\section{PRESENTACIÓN DEL CASO}

Hombre de 51 años de edad, sin antecedentes de importancia para el padecimiento actual. Presenta dolor abdominal en mesogastrio de inicio brusco tipo transfictivo, en hemicinturón con irradiación dorsal de intensidad 8/10, acompañado de náuseas, anorexia y vómito de contenido gastrointestinal en tres ocasiones, con exacerbación de dolor posprandial; niega fiebre y acolia, pero refiere coluria. A su ingreso: Leucocitos 19.2 $10^{3} / \mu \mathrm{L}$, neutrófilos $89.0 \%$, hemoglobina $13.5 \mathrm{~g} / \mathrm{dL}$, hematocrito $38.7 \%$, plaquetas $443.010^{3} / \mu \mathrm{L}$, procalcitonina $7.33 \mathrm{ng} / \mathrm{mL}$, aspartato aminotransferasa $138 \mathrm{UI} / \mathrm{L}$, alanina aminotransferasa $206 \mathrm{UI} / \mathrm{L}$, fosfatasa alcalina $335 \mathrm{UI} / \mathrm{L}$, gamma-glutamil transpeptidasa $271 \mathrm{UI} / \mathrm{L}$, bilirrubina total $6.61 \mathrm{mg} / \mathrm{dL}$, bilirrubina directa $4.18 \mathrm{mg} / \mathrm{dL}$, amilasa 767 UI/L, lipasa $3055 \mathrm{UI} / \mathrm{L}$.

La tomografía axial computarizada (TAC) muestra pancreatitis con Balthazar B, colecistitis aguda alitiásica. Colédoco dilatado hasta $8 \mathrm{~mm}$ sin evidencia de litos en su interior. Con diagnóstico de pancreatitis aguda leve Ranson 1, Bedside Index for Severity in Acute Pancreatitis (BISAP) 1 (Figura 1).

Se decide realizar colangiopancreatografía retrógrada endoscópica (CPRE) con diagnóstico de divertículo yuxtapapilar de $2 \times 2 \mathrm{~cm}$ de diámetro, divertículo duodenal a $5 \mathrm{~cm}$ de la papila hacia la tercera porción duodenal. Compresión extrínseca de colédoco distal secundaria a divertículo yuxtapapilar (Figura 2).

Se realiza esfinterotomía completa con abundante salida de bilis, sin detritus, se realizan dos barridos con balón

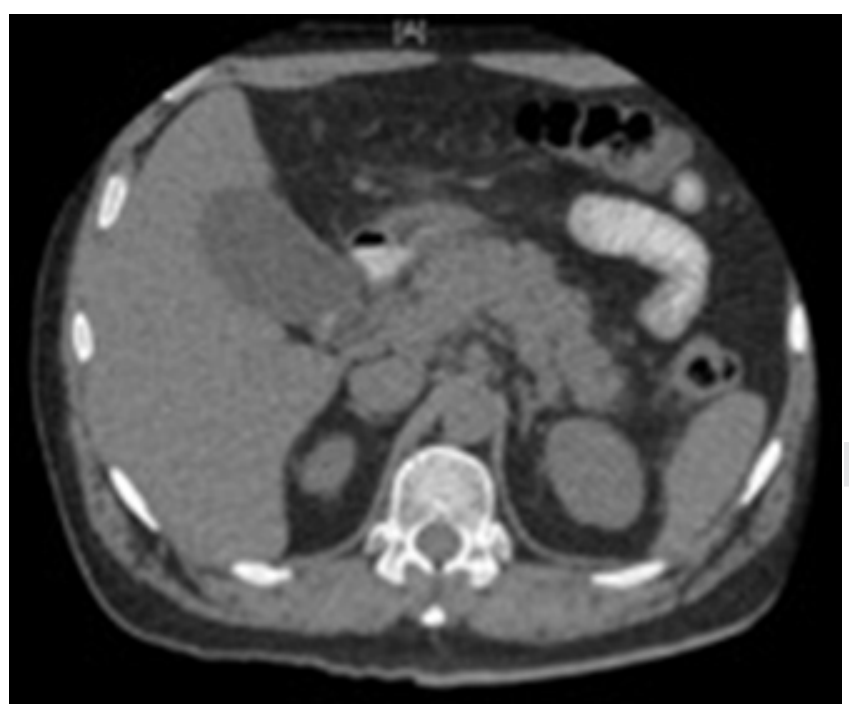

Figura 1: Pancreatitis aguda, clasificación Baltazar B. Colecistitis alitiásica.

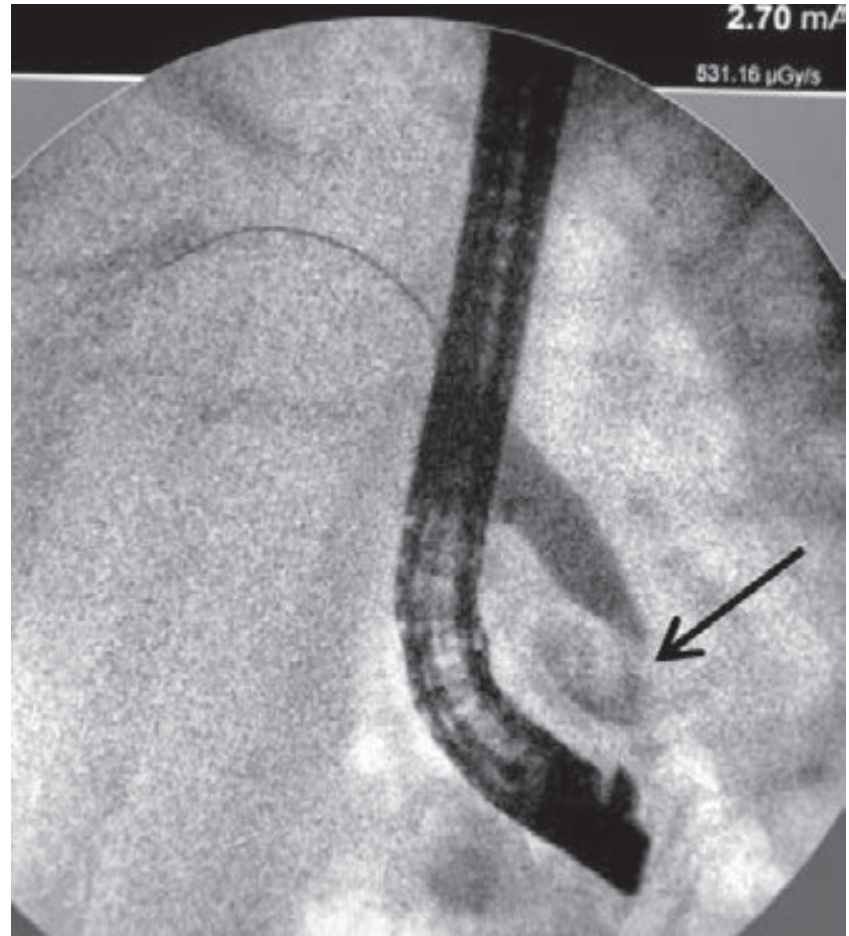

Figura 2: Colédoco distal comprimido por divertículo yuxtapapilar (flecha).

extractor sin observar salida de litos y se coloca prótesis plástica tipo Amsterdam.

Después del procedimiento, el paciente evoluciona de manera favorable con normalización de la biometría hemática y pruebas de función hepática (Figura 3).

En este caso el tratamiento endoscópico consistió en esfinterotomía y asegurar el drenaje biliar con endoprótesis, ya que por el divertículo yuxtapapilar aumenta el riesgo de presentar pancreatitis de repetición.

\section{DISCUSIÓN}

Por lo general, no se recomienda el tratamiento en pacientes asintomáticos. Los divertículos periampulares sintomáticos pueden ser tratados de manera conservadora, endoscópica o quirúrgica. ${ }^{6}$ El objetivo del tratamiento es aliviar la obstrucción biliopancreática mediante resección quirúrgica del divertículo, esfinterotomía endoscópica y/o endoprótesis. En la literatura se reporta el tratamiento endoscópico con la mayor tasa de éxito. ${ }^{7}$ El manejo quirúrgico se reserva hoy en día para complicaciones severas como perforación o hemorragia importante o en condiciones que cursen con sepsis severa, ${ }^{7}$ y la colangiopancreatografía retrógrada endoscópica con esfinterotomía es preferible en casos de alteraciones pancreatobiliares - como el caso de nuestro paciente con pancreatitis aguda-, la cual disminuye de manera considerable la morbimortalidad a com- 

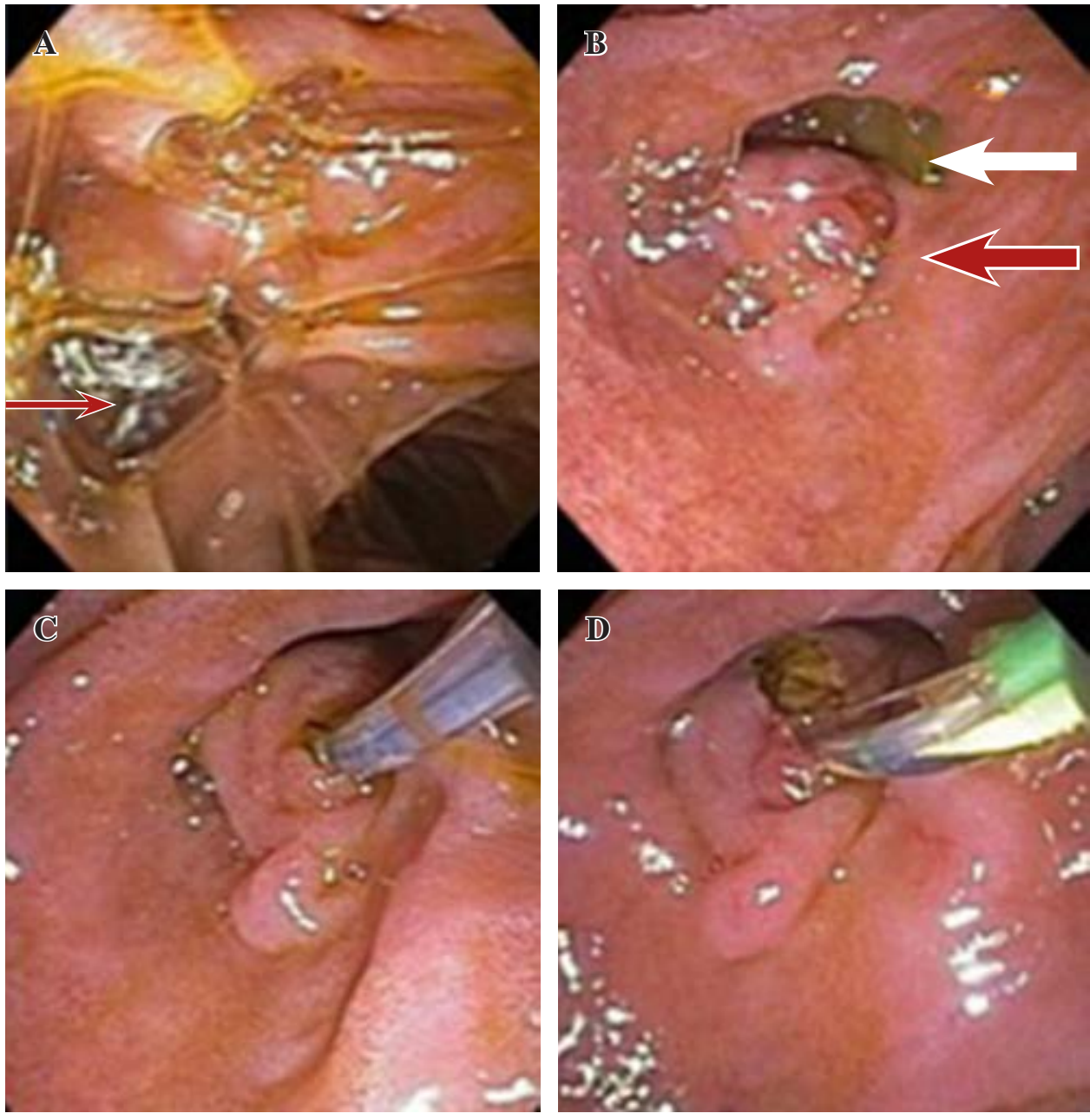

Figura 3:

A) Divertículo duodenal en tercera porción (flecha roja delgada). B) Divertículo yuxtapapilar flecha blanca gruesa) y ámpula de Vater (flecha roja gruesa). C y D) Tratamiento endoscópico con esfinterotomía completa.

paración de un evento quirúrgico. Por lo tanto, la colangiopancreatografía retrógrada endoscópica es el tratamiento de elección en pacientes sintomáticos en un centro hospitalario que cuente con el personal médico experto.

\section{CONCLUSIONES}

A pesar de que el síndrome de Lemmel no es conocido, se debe tomar en cuenta como etiología de obstrucción de la vía biliar no litiásica o neoplásica que puede manifestarse como ictericia obstructiva, colangitis o pancreatitis aguda, por lo que realizar el diagnóstico oportuno nos lleva a realizar una terapéutica correcta y así obtener los resultados favorables como en el caso reportado.

\section{REFERENCIAS}

1. Desai K, Wermers JD, Beteselassie N. Lemmel syndrome secondary to duodenal diverticulitis: a case report. Cureus. 2017; 9: e1066.
2. Khan BA, Khan SH, Sharma A. Lemmel's syndrome: a rare cause of obstructive jaundice secondary to periampullary diverticulum. Eur J Case Rep Intern Med. 2017; 4: 000632.

3. Mora-Guzmán I, Martín-Perez E. Lemmel's syndrome. Gastroenterol Hepatol. 2019; 42: 113-114.

4. Tham TC, Kelly M. Association of periampullary duodenal diverticula with bile duct stones and with technical success of endoscopic retrograde cholangiopancreatography. Endoscopy. 2004; 36: 1050-1053.

5. Schnueriger B, Vorburger SA, Banz VM, Schoepfer AM, Candinas D. Diagnosis and management of the symptomatic duodenal diverticulum: a case series and a short review of the literature. J Gastrointest Surg. 2008; 12: 1571-1576.

6. Beisani M, Espin F, Dopazo C, Quiroga S, Charco R. Manejo terapéutico del divertículo duodenal yuxtapapilar. Cir Esp. 2013; 91: 463-465.

7. Perdikakis E, Chryssou EG, Karantanas A. Diagnosis of periampullary duodenal diverticula: the value of new imaging techniques. Ann Gastroenterol. 2011; 24: 192-199. 